\title{
The Gamification User Types Hexad Scale
}

\author{
Gustavo F. Tondello1, Rina R. Wehbe1, Lisa Diamond², Marc Busch², \\ Andrzej Marczewski ${ }^{3}$, Lennart E. Nacke ${ }^{1}$ \\ ${ }^{1}$ HCI Games Group, Games Institute, University of Waterloo, Waterloo, ON, Canada \\ ${ }^{2}$ AIT - Austrian Institute of Technology GmbH, Vienna, Austria \\ ${ }^{3}$ Gamified UK, New Haw, Surrey, England \\ gustavo@tondello.com, rina.wehbe@uwaterloo.ca, lisa.diamond@ait.ac.at,marc.busch@ait.ac.at, \\ andrzej@gamified.uk, lennart.nacke@acm.org
}

\begin{abstract}
Several studies have indicated the need for personalizing gamified systems to users' personalities. However, mapping user personality onto design elements is difficult. Hexad is a gamification user types model that attempts this mapping but lacks a standard procedure to assess user preferences. Therefore, we created a 24-items survey response scale to score users' preferences towards the six different motivations in the Hexad framework. We used internal and testretest reliability analysis, as well as factor analysis, to validate this new scale. Further analysis revealed significant associations of the Hexad user types with the Big Five personality traits. In addition, a correlation analysis confirmed the framework's validity as a measure of user preference towards different game design elements. This scale instrument contributes to games user research because it enables accurate measures of user preference in gamification.
\end{abstract}

\section{Author Keywords}

Gamification; Gameful Design; User Types; Hexad.

\section{ACM Classification Keywords}

H.1.2. User/Machine Systems: Human Factors.

\section{INTRODUCTION}

Gamification, the use of game design elements in non-game contexts [12], has been operationalised to increase user engagement, activity, and enjoyment. Studies have shown that gamification can lead to positive behavioural changes; however, we currently do not understand the factors influencing user motivation in gamification. For example, Hamari et al. identified confounding factors such as the role of the context being gamified and the qualities of the users [17]. To better understand user motivation and to personalize the experience in gameful systems to each user, we propose a scale for preference assessment in gamification.

Permission to make digital or hard copies of all or part of this work for personal or classroom use is granted without fee provided that copies are not made or distributed for profit or commercial advantage and that copies bear this notice and the full citation on the first page. Copyrights for components of this work owned by others than the author(s) must be honored. Abstracting with credit is permitted. To copy otherwise, or republish, to post on servers or to redistribute to lists, requires prior specific permission and/or a fee. Request permissions from Permissions@acm.org.

CHI PLAY'16, October 16 - 19, 2016, Austin, TX, USA

Copyright is held by the owner/author(s). Publication rights licensed to ACM.

ACM 978-1-4503-4456-2/16/10 ..\$15.00

DOI: http://dx.doi.org/10.1145/2967934.2968082
Personalizing gameful systems to each user is important because personalized interactive systems are more effective than one-size-fits-all approaches. Gameful systems are effective when they help users achieve their goals, which often involve educating them about certain topics, supporting them in attitude or behaviour change, or engaging them in specific topics [9]. The efficacy of personalization according to the user's personality traits has been shown in user interface design [33], persuasive technology [24,25], and games $[1,34,35]$. As a consequence, we believe that personalized gameful systems will be more engaging if they adapt to personality traits or player types $[14,15]$.

Bartle's player typology for Multi-User Dungeons (MUDs) [3] is popularly used in gamification. However, it was created specifically for MUDs and it should not be generalized to other game genres nor to gameful design. To address this problem, Marczewski developed the Gamification User Types Hexad framework [27], based on research on human motivation, player types, and practical design experience. He also suggested different game design elements that may support different user types [28]. However, we still lack a standard assessment protocol for user's preferences based on the Hexad framework. There is also no empirical validation, yet, that associates Hexad user types and game design elements. In this paper, we address these two gaps.

Our work contributes to the field of gameful design ${ }^{1}$ in human-computer interaction (HCI) with two related goals. Firstly, we propose and validate a survey measure for scoring user's preferences towards different game design elements according to the Hexad framework. The questions were contributed by experts in scale development, game design, and HCI. We conducted an initial validation with 133 people, which confirmed the survey scale's reliability is within the acceptable limits. Next, we analyzed the correlations between the participants' scores in each of the Hexad user types with their scores on each Big Five personality trait as measured by the BFI-10 [38]. Positive correlations were found for the pairs in which the theoretical background suggested them, which also contributes to validate both the Hexad framework itself and the new survey scale.

\footnotetext{
${ }^{1}$ In this paper, we refer to gamification and gameful design indistinctively because they frame the same extension of phenomena through different intentional properties [12]. Thus, the Hexad model can be used for both.
} 
Secondly, we evaluate the potential of the Hexad framework as a model to personalize user experience (UX) in gameful systems. We asked participants to score their preferences regarding 32 design elements commonly employed in gameful design and analyzed their correlations with each of the Hexad user types. Overall, positive correlations were found between the Hexad user types and the corresponding game design elements, confirming the usefulness of the Hexad model to personalize gameful systems.

\section{RELATED WORK}

Understanding an individual's personality is a multifaceted endeavour. Theories of behaviour and personality are often employed to understand user behaviour and preferences in interactive systems because they provide some insight into motivating factors. Especially, Self-Determination Theory (SDT) $[10,40]$ provides the theoretical background for the Hexad model concerning the expression of both intrinsic and extrinsic motivation. Thus, the Hexad user types are expressions of these distinct motivating factors.

\section{Motivation}

Within HCI research, the principles of SDT [10,40-42] are often used as an explanation to provide insights into behaviour motivation. SDT suggests that individual motivation to engage in a task can be located within a range of different grades of internalization. In a simplified model, motivation can be intrinsic, i.e., afforded by the individual's perception of a task as enjoyable by itself, or extrinsic, i.e., afforded by factors outside of the task, such as expected outcomes that may result from completing the task.

Intrinsic motivation is supported in SDT by three components. Competence marks the feeling of having the skills needed to accomplish the task at hand. Autonomy means the more in control of a situation a person feels, the more likely they are to succeed. Finally, relatedness is the feeling of involvement with others. Additional work in the field by Ryan et al. [39] notes the importance of these three pillars and indicates that they can strongly contribute to a person's mental health benefits. Furthermore, the Hexad model is also informed by the evidence that meaning (purpose) facilitates internalization, increasing the motivation to carry out uninteresting but important activities [11], and leads to increased happiness and life satisfaction [20,37].

\section{Personality}

Previous research has demonstrated that personality has an effect on player types [32] and player preferences of different game genres [23] and gamification elements [21]. Personality also seems to affect how players experience psychological satisfaction in games [22] and presence in virtual reality applications [26]. Thus, we decided to study how different personality traits relate to the Hexad user types.

A common way to analyse people's personalities is via the five-factor model of personality, commonly known as the Big Five. The Big Five provides a survey measure of five main categories of personality factors. Openness referring to an adventure seeking or an open-to-experience person;
Conscientiousness is related to thought and organization; Extraversion or outgoing personality and Agreeableness referring to the qualities associated with the person's relation to others; and finally, Neuroticism or level of selfsecurity and confidence. We used a short, ten-questions version of the Big Five survey scale (BFI-10, [38]).

Johnson and Gardner [22] have previously studied the relationship between the Big Five personality traits and the fulfillment of psychological needs in video games. They found positive correlations between agreeableness and competence; and openness to experience and autonomy; as well as a negative correlation between emotional stability and presence. Yee et al. [45] studied how personality traits affect player behaviour in World of Warcraft. They related extraversion with the preference for group activities, agreeableness with more frequent use of emotes and preference for non-combat activities, conscientiousness with the enjoyment of disciplined collections in non-combat settings, neuroticism with a preference for Player vs. Player activities, and openness with curiosity-driven gameplay, such as creating new characters or exploring the game world. Jia et al. [21] studied the correlation between the Big Five personality traits and individual gamification affordance elements. They found positive correlations of extraversion with points, levels, and leaderboards; agreeableness with challenges; and conscientiousness with levels and progress; as well as negative correlations of emotional stability (the opposite of neuroticism) with points, badges, progress, and rewards; and openness with avatars.

The Balanced Inventory of Desirable Responding (BIDR) [36] is also often used in scale validation to evaluate people's tendency to bias their self-reported answers in a socially desirable way. The BIDR measures two constructs: self-deceptive enhancement (SDE), or the tendency to give reports that are honest but positively biased, and impression management (IM), or the tendency to deliberately construct a self-presentation to an audience. We used a short, sixquestions version of the scale (BIDR-6, [43]).

\section{Personalization in Games and Gameful Design}

Personalization can be used in game design to tailor game mechanics to the player or in gameful design to tailor interaction mechanics to the user. Using player or user typologies to understand individual preferences is one of the common approaches for personalization. Thus, several different models exist in the literature. We review some of these most well-known and recent models, which informed the creation of the Hexad model. A more comprehensive review has been done by Hamari and Tuunanen [18].

One of the oldest and most frequently used player type models is Bartle's player type model [3] and its extensions [4]. Bartle identified four player types (Achiever, Explorer, Socialiser, and Killer) for players of Multi-User Dungeons (MUDs) based on what they desired from a MUD.

Yee $[46,47]$ used a factor analysis approach out of questions based on Bartle's player types. His analysis identified 
three main components of player motivation with ten subcomponents: achievement (advancement, mechanics, competition), social (socializing, relationship, teamwork), and immersion (discovery, role-playing, customization, escapism). Like Bartle's model, Yee's components have a strong focus on one specific game genre, Massively Multiplayer Online Role-Playing Games (MMORPGs). It was not created or intended for a broad range of different game genres.

A wider perspective regarding player types is included in the first Demographic Game Design model (DGD1) [6], which is an adoption of the Myers-Briggs Type Indicator (MBTI, [30]) to games. It proposed the player styles Conqueror, Manager, Wanderer, and Participant. The second Demographic Game Design model (DGD2) [5] explored the hard-core to casual dimension, different skill sets, and the preference for single and multiplayer. Although providing valuable insights into player characteristics, both DGD1 and DGD2 are based on a pre-existing psychometric model (MBTI) that is not focused on games. The authors also reported issues related to methodology and data collection.

Emerging from an empirical evaluation of a health game for younger adults, Xu et al. [44] developed five player types: achievers, active buddies, social experience seekers, team players, and freeloaders. These types include both motivational and behavioural factors. However, they have not been investigated regarding their validity to personalize games.

The BrainHex model [31,32] was developed considering previous player typologies and neurobiological research. It introduces seven player archetypes: Achiever, Conqueror, Daredevil, Mastermind, Seeker, Socialiser, and Survivor. It is a promising approach, supplementing existing research with a more diverse array of player types, and it has been initially investigated on its psychometric properties [8] and been used in a number of recent studies in HCI $[7,35,48]$.

While these models are often used in personalizing gameful systems, they were built specifically for game design, thus, their usefulness for gameful design is limited.

Looking at models created specifically for gameful design, Barata et al. [2] studied data regarding student performance and gaming preferences from a gamified university level engineering course and identified four student types related to different gaming preferences: Achievers, Regular Students, Half-hearted Students, and Underachievers.

Barata's model is specific to the domain of gamified learning. Differently, the Hexad model aims at covering a broad range of gameful systems. Therefore, we consider the Hexad model to be potentially suitable for personalization of gameful systems and thus warrant further research.

\section{Gamification User Types Hexad}

Marczewski proposed six user types that differ in the degree to which they can be motivated by either intrinsic (e.g., self-realization) or extrinsic (e.g., rewards) motivational factors [27]. Rather than basing the model on observed behaviour, the user types are personifications of people's

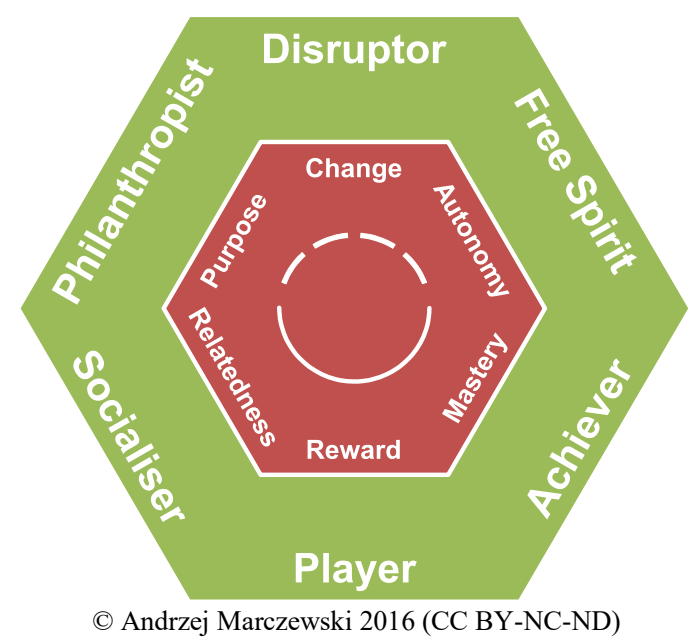

Figure 1. Gamification User Types Hexad [27].

intrinsic and extrinsic motivations, as defined by SDT [41]. Accordingly, the four intrinsically motivated types in the Hexad model are derived from the three types of intrinsic motivation from SDT, namely relatedness, competence, and autonomy, with the addition of purpose [11]. Figure 1 illustrates the six user types from the Hexad model. Below, we list the user types and the game design elements suggested by Marczewski to address the motivations of each type [28], which we investigate in this work.

Philanthropists are motivated by purpose. They are altruistic and willing to give without expecting a reward.

Suggested design elements: collection and trading, gifting, knowledge sharing, and administrative roles.

Socialisers are motivated by relatedness. They want to interact with others and create social connections.

Suggested design elements: guilds or teams, social networks, social comparison, social competition, and social discovery.

Free Spirits are motivated by autonomy, meaning freedom to express themselves and act without external control. They like to create and explore within a system.

Suggested design elements: exploratory tasks, nonlinear gameplay, Easter eggs, unlockable content, creativity tools, and customization.

Achievers are motivated by competence. They seek to progress within a system by completing tasks, or prove themselves by tackling difficult challenges.

Suggested design elements: challenges, certificates, learning new skills, quests, levels or progression, and epic challenges (or "boss battles").

Players are motivated by extrinsic rewards. They will do whatever to earn a reward within a system, independently of the type of the activity.

Suggested design elements: points, rewards or prizes, leaderboards, badges or achievements, virtual economy, and lotteries or games of chance. 
Disruptors are motivated by the triggering of change. They tend to disrupt the system either directly or through others to force negative or positive changes. They like to test the system's boundaries and try to push further. This type is derived from SDT, but from empirical observation of this behaviour within online systems. Although disruption can sometimes be negative (e.g., cheaters or griefers), this is not always the case because disruptors can also work to improve the system.

Suggested design elements: innovation platforms, voting mechanisms, development tools, anonymity, anarchic gameplay.

Some motivations underlying these user types are related, but the user types themselves overlap slightly. Achievers and Players are both motivated by achievement, but differ in their focus: Players focus on extrinsic rewards while Achievers focus on competence. Philanthropists and Socialisers are both motivated to interact with other players. However, they differ because a Socialiser's interest is in the interaction itself while a Philanthropist is motivated by interaction to help others. Finally, Free Spirits and Disruptors are both motivated by autonomy and creativity. However, Free Spirits stay within the system limits without a desire to change them and Disruptors seek to expand beyond these boundaries to change the system.

It is also worth noting that, although these motivation clusters are presented as user types, individuals are rarely motivated by one of them exclusively. Although users are likely to display a principal tendency, in most cases they will also be motivated by all the other types to some degree.

There are several ways to use the Hexad model to personalize gameful applications. For example, Jia et al. [21] provide design suggestions for gameful applications based on the Big Five traits, but the Hexad model has even more potential for customizing gameful applications since it is modeled after player motivations specific to gameful applications. Designers would be able to screen their target audience using the suggested survey and choose the adequate design elements for each user. In research, the survey can be used to better understand user engagement and enjoyment in studies regarding gameful applications.

\section{METHODOLOGY}

We conducted our work in three sequential phases:

1. survey scale construction, in which we developed a standard survey response scale for the Gamification User Types Hexad framework;

2. data collection, in which we collected responses from an on-line survey with questions related to the Hexad framework, preferences regarding game design elements, and personality tests;

3. data analysis, in which we analyzed the responses to accomplish two goals: validate the User Types Hexad scale and evaluate the potential of the Hexad framework to personalize the user experience in gameful systems.

\section{Survey Scale Construction}

To design the Gamification User Types Hexad Scale, we followed a systematic approach involving an expert workshop to generate items based on the available framework and a subsequent expert validation process to evaluate and rate items based on their face validity. The new scale is inspired by but is not the same as the previous attempt by Marczewski to create an assessment tool [29], which did not follow a systematic approach and was found to be unreliable. The survey construction phase has already been described in more detail elsewhere [13].

We started the development of the survey with an expert workshop conducted by the Austrian Institute of Technology to generate a pool of items for each of the different Hexad user types. A group of six experts with either an expertise in scale development or game mechanics was introduced to the Gamification User Types Hexad framework [27] through detailed material, explaining each of the different types and the game mechanics they are likely to respond to as suggested by Marczewski [28]. Subsequently, each expert was asked to develop a list of items that would describe each of the user types. Each item aimed to help assess the participant's inclination towards one of the user types. Once this task was completed, the developed items were pooled successively for each type and discussed. As part of these discussions, the defining characteristics were reviewed and the created item pools were extended to cover missing aspects of the respective types as needed.

As the second step of the survey development, we reviewed the list of items created for each type, removing those items that seemed misleading, too broad, too context-dependent, or redundant. A rating form was created for the remaining list of 74 items. We then sent the form out for an expert rating by the group of experts involved in the workshop, as well as to the creator of the Hexad framework, Andrzej Marczewski, and two experts in the fields of HCI and games. The expert jury was asked to judge how well each item represented its gamification user type (along a 6-point scale ranging from "very bad" to "very good") and to comment if any potential issues were observed (e.g., an item insufficiently differentiating between related types). Further, everyone was invited to mention any aspects of the player types they observed as not sufficiently covered. Once all rating forms were returned, we analyzed the ratings (mean, range). Then, we selected the items with the best ratings for a first 30 -item version of the survey. The list of survey items is included in the appendix.

\section{Survey Instrument}

We conducted an online survey with students of the University of Waterloo, Canada, which was completed in English and contained the following sections:

1. Demographic information: age, gender, education level, native country, native language, and self-reported level of English proficiency (to identify possible misunderstandings because of lacking language proficiency). 
2. Hexad User Types survey items: we asked participants to rate the 30 items related to the six Hexad user types (see appendix) on a 7-point Likert scale and to comment on the items (e.g., to mention any item they found confusing or hard to understand).

3. Game Elements preferences: we asked participants to rate how much they are motivated by 32 different game design elements (as used indistinctively in games or gameful applications) on a 7-point Likert scale.

4. Personality: the BIDR-6 [43] and the BFI-10 [38] personality surveys using 7-point Likert scales were included to gain insight into potential relationships between user types and personality.

We asked participants for permission to contact them again in a few weeks to answer a follow-up survey aimed at enabling calculation of a test-retest reliability score for the Hexad User Types survey. The follow-up survey contained only the Hexad User Types question section.

\section{Participants}

One hundred and thirty-three graduate and undergraduate students from the University of Waterloo, Canada (64 females, 59 males, 10 declined to answer), aged 18-36 ( $M=$ 23.5 years, $S D=3.3$ years), volunteered to participate in the online survey. From those 133 respondents, 40 participated in the follow-up survey. They were recruited through the University's mailing lists and bulletin boards and offered a chance to win a CAD\$ 50.00 (approximately USD\$ 38.00) Amazon gift card in a draw after completing the survey. Informed consent was obtained from all participants. Answers were collected in March 2016. Retest data were collected two weeks after the initial survey.

Regarding English language proficiency, 75 participants reported having English as a native language and 58 reported a variety of different native languages. However, 90 participants reported a native English proficiency level (i.e., some considered themselves as proficient as a native, even not being a native speaker), 36 reported a very good proficiency, and four reported a fair proficiency (three declined to answer). Thus, we operate on the assumption that lack of English proficiency was not a detriment to our study.

\section{Analytical Procedure}

We conducted our data analysis in three steps: scale reliability, scale correlation with personality traits, and scale correlation with game design elements. In all cases, we used Kendall's $\tau$ to calculate correlations, following Howell's suggestion that it provides the best estimates for nonparametric data [19], as the scores were not normally distributed. However, doing so requires attention to effect size interpretation because the absolute value of $\tau$ is usually lower than the values of the more commonly known Pearson's $r$ and Spearman's $\rho$ for the same effect sizes [16]. Therefore, we used the correspondence tables calculated by Gilpin [16] to interpret Kendall's $\tau$ effect size according to the approximate Pearson's $r$ equivalent:
- small effect: $\quad \tau=0.20 \quad(\approx r=0.30)$;

- medium effect: $\tau=0.34 \quad(\approx r=0.50)$;

- large effect: $\tau=0.50 \quad(\approx r=0.70)$.

Scale Reliability

We analyzed the scale's internal reliability by separately calculating the Cronbach's $\alpha$ for each one of the six subscales. In addition, we evaluated the individual contribution of each item to its subscale and the participants' comments to decide whether to keep or remove less reliable items. After verifying the scale's reliability, we calculated each participant's score for each of the six Hexad types as the median of the rates reported by the participant for each of the items composing the subscale. These scores were employed for the subsequent tests. To evaluate the test-retest reliability, we separately calculated the bivariate correlation between the original and the retest scores of each subscale using Pearson's $r$.

Next, we calculated the bivariate correlation coefficients of each user type with all others using Kendall's $\tau$. Considering the Hexad model's theoretical background regarding overlapping user types, we expected the following significant correlations: Achiever with Player, Philanthropist with Socialiser, and Free Spirit with Disruptor.

Finally, we tested the bivariate correlation of each subscale's score with the two scales from the BIDR-6 using Kendall's $\tau$, to verify if participants' responses could have been influenced by a tendency to desirable responding.

\section{Scale Correlation with Personality Traits}

First, we analyzed the bivariate correlation between the Big Five personality traits and the BIDR-6 scales to control for acquiescence regarding the BFI-10 scale. We do not present the full results table due to space limitations. We found a significant strong negative correlation between neuroticism and $\operatorname{SDE}(\tau=-0.438, p<0.01)$; therefore, participants' scores on neuroticism might be underestimated by their tendency to protect their self-esteem. We then analyzed the bivariate correlations between the Hexad scale and the Big Five model of personality traits by separately calculating the correlation coefficient between each pair of Hexad type and personality trait using Kendall's $\tau$. Based on previous literature $[21,22,45]$, we establish the following hypotheses:

H1: The Achiever user type is positively correlated with agreeableness and conscientiousness.

H2: The Free Spirit user type is positively correlated with openness to experience.

H3: The Player user type is positively correlated with extraversion and neuroticism.

In addition, - although this correlation did not appear previously - we can also make the following hypothesis because the Socialiser and Philanthropist types are both based on social interactions:

H4: The Philanthropist and Socialiser user types are positively correlated with extraversion. 


\section{Scale Correlation with Game Design Elements}

To analyze the correlation of the Hexad types with the set of game design elements, we firstly calculated scores for six sets of game elements, corresponding to the six Hexad types, following the division suggested by Marczewski [28]. We then separately calculated the bivariate correlation between each pair of Hexad type with its corresponding set of game design elements using Kendall's $\tau$.

After initial analysis, we calculated the bivariate correlation of each individual game design element with each of the six Hexad types using Kendall's $\tau$. This was done to evaluate the distribution of the design elements between the Hexad user types and to suggest improvements based on reported preferences. Next, we suggested a new association table between design elements and user types. As a general rule, correlations with a coefficient $\tau>0.20$ were considered meaningful because this represents the threshold for a small-sized correlation. After creating the new table, we recalculated the scores for each element set and compared them to the original scores to verify improvements.

\section{RESULTS}

Our analysis included the following measures, as reported in the Methodology section: scale reliability, distribution of the user types scores, scale correlation with personality traits, and scale correlation with game design elements.

\section{Scale Reliability}

Table 1 presents the internal reliability coefficients of each of the subscales, measured by Cronbach's $\alpha$. After evaluating each subscale's reliability, we analyzed the individual reliability of each item. Two items were found to poorly contribute to their scales: "I look out for my own interests" (Player) and "I like to take changing things into my own hands" (Disruptor). Moreover, a few participants reported that they could not precisely understand the meaning of the latter Disruptor item. Thus, we removed these two items. We then analyzed the effect of removing the least reliable item of each of the remaining four subscales on their reliabilities. The effects were small $(<=0.008)$. Thus, we removed the least reliable subscale items, arriving at a final scale with 24 items (four items per subscale). The recalculated internal reliability coefficients for the 24-items scale are presented in Table 1 . In addition, the scale correlations for all items are presented later in Table 8.

\begin{tabular}{r|rr} 
User Type & 5-items subscale & 4-items subscale \\
\hline Philanthropist & 0.896 & 0.893 \\
Socialiser & 0.846 & 0.838 \\
Free Spirit & 0.727 & 0.723 \\
Achiever & 0.766 & 0.759 \\
Disruptor & 0.728 & 0.738 \\
Player & 0.689 & 0.698
\end{tabular}

Table 1. Internal scale reliability (Cronbach's $\alpha$ ) for each of the Hexad User Types in the original 30-items (5 per subscale) and the final 24 -items (4 per subscale) surveys.
Table 2 exhibits the test-retest correlation coefficients for each of the subscales, measured by Pearson's $r$. All subscales presented a high test-retest reliability for the 24-items scale, except for the Player type, which presented a small coefficient. Moreover, results show that removing the least reliable item per subscale represent a small overall improvement in test-retest reliability.

Table 3 presents the bivariate correlation coefficients and significance levels between each Hexad user type and the BIDR subscales. Results show significant weak correlations between Free Spirit, Achiever, and Player with SDE and between Philanthropist and Disruptor with IM (the latter is negative). This seems to suggest that the achievement- and autonomy-oriented subscales might be just slightly overestimated by participants' tendency to protect their selfesteem; and that the philanthropist subscale might be slightly overestimated and the disruptor subscale slightly underestimated by participants' desire to please others.

Table 4 presents the correlation coefficients between each user type and all others. Positive medium-sized correlations were found between the pairs suggested by the theoretical background: Philanthropist with Socialiser, Free Spirit with Disruptor, and Achiever with Player. Furthermore, similar magnitude correlations were also found between Philanthropist and Free Spirit, Achiever and Free Spirit, and Player and Free Spirit. Other significant correlations that appeared were of weaker magnitude.

We also conducted a factor analysis with a maximumlikelihood method and an Oblimin rotation with Kaiser

\begin{tabular}{r|rr} 
User Type & 5-items subscale & 4-items subscale \\
\hline Philanthropist & $0.850^{* *}$ & $0.852^{* *}$ \\
Socialiser & $0.820^{* *}$ & $0.853^{* *}$ \\
Free Spirit & $0.483^{* *}$ & $0.631^{* *}$ \\
Achiever & $0.752^{* *}$ & $0.798^{* *}$ \\
Disruptor & $0.611^{* *}$ & $0.782^{* *}$ \\
Player $^{*} p<0.05 . \quad{ }^{* *} p<0.01$. & $0.387^{*}$ & $0.357^{*}$
\end{tabular}

Table 2. Test-retest reliability (Pearson's $r$ ) for Hexad User Types in the original 30-items (5 per subscale) and the final 24items (4 per subscale) surveys.

\begin{tabular}{r|rc} 
User Type & S.D.E. & I.M. \\
\hline Philanthropist & $0.066^{*}$ & $\mathbf{0 . 1 3 5}$ \\
Socialiser & $0.042^{* *}$ & 0.060 \\
Free Spirit & $\mathbf{0 . 2 0 4}{ }^{* *}$ & 0.065 \\
Achiever & $\mathbf{0 . 2 0 9}{ }^{* *}$ & $0.084^{* *}$ \\
Disruptor & $0.021^{*}$ & $\mathbf{- 0 . 1 7 3}$ \\
Player & $\mathbf{0 . 1 4 6}$ & 0.003
\end{tabular}

Table 3. Bivariate correlation coefficients (Kendall's $\tau$ ) and significance between Hexad user types and desirable responding sub-scales: self-deception enhancement (S.D.E.) and impression management (I.M.). 


\begin{tabular}{r|rrrrr} 
User Type & Philanthropist & Socialiser & Free Spirit & Achiever & Disruptor \\
\hline Socialiser & $\mathbf{0 . 4 7 6}$ & & & \\
Free Spirit & $\mathbf{0 . 3 2 8}^{* *}$ & $0.274^{* *}$ & & & \\
Achiever & $\mathbf{0 . 3 2 5}$ & $0.218^{* *}$ & $\mathbf{0 . 4 6 5}^{* *}$ & & \\
Disruptor & $-0.057^{* *}$ & $-0.001^{* *}$ & $\mathbf{0 . 2 4 9}$ & 0.045 & \\
Player $^{* *} p<0.01 . \quad$ & $0.213^{* *}$ & $\mathbf{0 . 3 0 7}^{* *}$ & $\mathbf{0 . 3 5 4}^{* *}$ & $\mathbf{0 . 4 3 2}$ & \\
& & & &
\end{tabular}

Table 4. Bivariate correlation coefficients (Kendall's $\tau$ ) and significance between each Hexad user type and all others.

normalization. We forced a six factors analysis to evaluate the correspondence of the factors with the Hexad user types. We report the rotated factor loads later in Table 8. We report only factor loads higher than 0.20 to improve readability. Together, the six factors explained $55.1 \%$ of the variance in the data. The results show that the six factors correspond to the Hexad types in overall, with some overlapping in the Philanthropist, Free Spirit, and Achiever types, which correspond to the overlaps found between the different user types (see Table 4).

\section{Distribution of the User Types Scores}

Table 5 presents the mean and standard deviation of the calculated scores for each of the six user types from the 24items scale. For better readability, scores are presented as the sum of each item's rates instead of the mean (i.e., the maximum value for each subscale is 28). A visual inspection reveals that the average score for the Disruptor type is considerably lower than the other types. Figure 2 presents the distribution of participants' main Hexad User Type, i.e., the type in which the participant achieved the highest score. These data suggest that the four user types based on intrinsic motivation - Philanthropist, Socialiser, Free Spirit, and Achiever - are similarly common as the main user type, while the Player type is also somewhat common as the main user type, but half as common as the intrinsic types, and the Disruptor type is not at all common as the main user type.

\section{Scale Correlation with Personality Traits}

Table 6 presents the bivariate correlations coefficients and significance levels between each Hexad user type and each of the Big Five model personality traits, measured by Kendall's $\tau$. The Philanthropist type is positively correlated with extraversion, supporting hypothesis H4, and also with agreeableness, conscientiousness, and openness. The Socialiser type is positively correlated with extraversion, supporting hypothesis H4, and also with agreeableness. The Free Spirit type is positively correlated with openness, sup-

\begin{tabular}{r|rr} 
User Type & Mean Score & S.D. \\
\hline Philanthropist & 22.36 & 4.72 \\
Socialiser & 20.33 & 5.09 \\
Free Spirit & 22.09 & 4.06 \\
Achiever & 22.18 & 3.97 \\
Disruptor & 14.94 & 4.80 \\
Player & 20.99 & 4.08
\end{tabular}

Table 5. Average scores and SD for each Hexad user type. porting hypothesis H2, and also positively correlated with extraversion and negatively with neuroticism. The Achiever type is positively correlated with conscientiousness; however, it was not found to be correlated with agreeableness. Thus, hypothesis $\mathbf{H 1}$ is only partly supported. This appears to differ from Johnson and Gardner's findings [22]. However, they measured how much players felt competent after gameplay, while our survey scores general user preferences towards different game design elements. Therefore, while both measures are based on the intrinsic need for competence, they are not measuring the same effect. This fact might explain the contradiction. The Disruptor type is negatively correlated with neuroticism. Finally, the Player type is only positively correlated with conscientiousness, thus, hypothesis $\mathbf{H 3}$ was not supported. In summary, our results lead to the following conclusions:

H1: partly supported. The Achiever user type was positively correlated with conscientiousness, but not with agreeableness.

H2: supported. The Free Spirit user type was positively correlated with openness to experience.

H3: not supported. The Player user type was not correlated with either extraversion or neuroticism.

H4: supported. The Philanthropist and Socialiser user types were positively correlated with extraversion.

Furthermore, there are three sets of correlations that were not hypothesized but raised from the data: Philanthropist and Socialiser with agreeableness; Philanthropist and Player with Conscientiousness; and Free Spirit and Disruptor with emotional stability (the opposite of neuroticism).

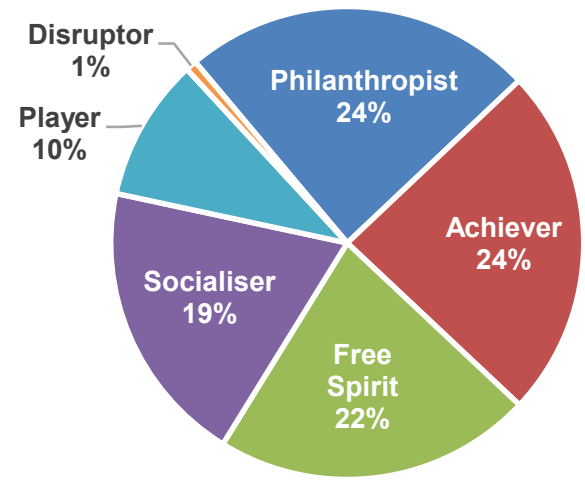

Figure 2. Distribution of participants' Hexad User Type. 


\begin{tabular}{|c|c|c|c|c|c|}
\hline User Type & Extraversion & Agreeableness & Conscientiousness & Neuroticism & Openness \\
\hline Philanthropist & 0.148 * & $0.191^{* *}$ & 0.159 * & 0.013 & 0.145 * \\
\hline Socialiser & $0.290^{* *}$ & $0.272^{* *}$ & 0.079 & -0.073 & 0.082 \\
\hline Free Spirit & $0.152 *$ & 0.089 & 0.078 & $-0.204^{* *}$ & $0.215^{* *}$ \\
\hline Achiever & -0.005 & 0.041 & $0.255^{* *}$ & -0.117 & 0.027 \\
\hline Disruptor & 0.038 & -0.106 & -0.080 & $\mathbf{- 0 . 1 7 0}{ }^{* *}$ & 0.090 \\
\hline Player & 0.054 & 0.121 & $0.144^{*}$ & -0.054 & 0.093 \\
\hline
\end{tabular}

${ }^{*} p<0.05$. $\quad{ }^{* *} p<0.01$.

Table 6. Bivariate correlation coefficients (Kendall's $\tau$ ) and significance of Hexad user types with Big Five personality traits.

Scale Correlation with Game Design Elements

Table 7 presents the game design elements suggested to address the motivations of each user type, together with the correlation coefficients between each user type's mean score and the corresponding design elements' mean score per participant. Overall, the user types were positively correlated with the corresponding game design elements, which confirms the validity of the Hexad model for understanding user preferences towards different elements. The exception was the Philanthropist type, for which no correlation was found with the expected design elements.

We do not present the complete correlation table of the individual game design elements because of space constraints, but it is included in the appendix. The analysis revealed that some design elements presented a higher correlation coefficient with a different user type than that suggested by Marczewski [28] or were correlated with more than one user type. In addition, some design elements only presented insignificant or weak correlations. Thus, we suggest a new association table between user types and game design elements considering, as a general rule, the significant correlations with a coefficient higher than 0.20 (weak or stronger correlations). However, we cannot make a suggestion for the Philanthropist type because we did not encounter design elements significantly correlated with it. Table 7 also exhibits the new association table and the new correlation coefficients after the adjustment. Design elements that were correlated with more than one user type are shown as Additional Elements. Moreover, we compare the new correlation coefficients with the previous ones to measure the improvement. Results show that our new suggested association table leads to an overall improvement of $9 \%$ over the table suggested by Marczewski.

\section{DISCUSSION}

In this section, we discuss the implications of our findings.

\section{Scale Reliability}

After evaluating the internal reliability of each subscale, we arrived at a final 24-items survey that represented the optimal format (see Table 8 and the appendix). Results showed a need to improve the Player subscale in future work as both its internal $(0.698)$ and test-retest $(0.357)$ reliabilities were below the desired levels. All other scales achieved the desired reliability, as it was higher than 0.70 for Free Spirit, Achiever, and Disruptor, and higher than 0.80 for Philanthropist and Socialiser.
The correlation analysis between the Hexad user types and the BIDR showed only weak correlations of Free Spirit, Achiever, and Player with self-deceptive enhancement and of Philanthropist and Disruptor with impression management. This is relevant for future work, but this effect was not strong enough to be considered influential to the results.

The correlation analysis of the Hexad user types between themselves showed positive correlations where suggested by the theoretical background (Philanthropist with Socialiser, Free Spirit with Disruptor, and Achiever with Player). Furthermore, we found unexpected correlations between Philanthropist and Free Spirit, Achiever and Free Spirit, as well as Player and Free Spirit, which suggest avenues for further investigation. The factor analysis corroborates these findings and confirms that the scale items correspond to their nominal subscales, with some partial overlaps.

These results collectively confirm that the Hexad user types can be measured empirically and correspond to the expected effects according to their theoretical background. Thus, we reinforce the relevance of the Hexad model for future developments in gameful design and HCI.

\section{Scale Correlation with Personality Traits}

Analysis of the correlations between the Hexad user types and personality traits showed significant correlations on most of the pairs where they were suggested by the theoretical background, further contributing to validate the Hexad model. Positive correlations were found of Philanthropist and Socialiser with extraversion, Achiever with conscientiousness, and Free Spirit with openness. Moreover, we found unexpected correlations of Philanthropist and Socialiser with agreeableness, which can be explained by the fact that all are connected with social relations, and of Achiever and Player with conscientiousness, which can help explain their orientation towards goals and rewards. Additionally, Free Spirit and Disruptor were correlated with emotional stability, which seems counter-intuitive. This can be partially explained by a potential deviation on the scores for neuroticism due to self-deceptive enhancement. Thus, these results suggest interesting avenues for further investigation.

\section{Scale Correlation with Game Design Elements}

We found positive correlations between all Hexad user types with the expected game design elements, except Philanthropist. These results validate the usefulness of the Hexad framework as a tool to personalize gamified 


\begin{tabular}{|c|c|c|c|c|c|c|}
\hline \multirow[b]{2}{*}{ User Type } & \multicolumn{2}{|c|}{ Suggested by Marczewski [28] } & \multicolumn{3}{|c|}{ Improved Associations } & \multirow[b]{2}{*}{ Imp. } \\
\hline & Design Elements & $\tau$ & Principal Elements & Additional Elements & $\tau$ & \\
\hline Philanthropist & $\begin{array}{l}\text { Collection and Trading } \\
\text { Gifting } \\
\text { Knowledge Sharing } \\
\text { Administrative Roles }\end{array}$ & 0.039 & 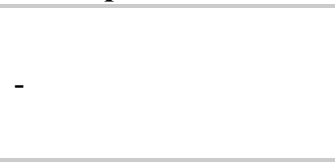 & & - & - \\
\hline Socialiser & $\begin{array}{l}\text { Guilds or Teams } \\
\text { Social Networks } \\
\text { Social Comparison } \\
\text { Social Competition } \\
\text { Social Discovery }\end{array}$ & $0.257^{* *}$ & $\begin{array}{l}\text { Guilds or Teams } \\
\text { Social Networks } \\
\text { Social Comparison } \\
\text { Social Competition } \\
\text { Social Discovery }\end{array}$ & & $0.257^{* *}$ & $0 \%$ \\
\hline Free Spirit & $\begin{array}{l}\text { Exploratory Tasks } \\
\text { Nonlinear Gameplay } \\
\text { Easter Eggs } \\
\text { Unlockable Content } \\
\text { Creativity Tools } \\
\text { Customization }\end{array}$ & $0.341^{* *}$ & $\begin{array}{l}\text { Exploratory Tasks } \\
\text { Nonlinear Gameplay } \\
\text { Easter Eggs } \\
\text { Unlockable Content } \\
\text { Learning } \\
\text { Anonymity } \\
\text { Anarchic Gameplay }\end{array}$ & $\begin{array}{l}\text { Customization } \\
\text { Challenges } \\
\text { Creativity Tools }\end{array}$ & $0.386^{* *}$ & $13 \%$ \\
\hline Achiever & $\begin{array}{l}\text { Challenges } \\
\text { Certificates } \\
\text { Learning } \\
\text { Quests } \\
\text { Levels or Progression } \\
\text { Epic Challenges }\end{array}$ & $0.347^{* *}$ & $\begin{array}{l}\text { Challenges } \\
\text { Certificates } \\
\text { Quests }\end{array}$ & $\begin{array}{l}\text { Anonymity } \\
\text { Learning } \\
\text { Badges or Achiev. } \\
\text { Levels or } \\
\quad \text { Progression }\end{array}$ & $0.362^{* *}$ & $4 \%$ \\
\hline Disruptor & $\begin{array}{l}\text { Innovation Platforms } \\
\text { Voting Mechanisms } \\
\text { Development Tools } \\
\text { Anonymity } \\
\text { Anarchic Gameplay }\end{array}$ & $0.326^{* *}$ & $\begin{array}{l}\text { Innovation Platforms } \\
\text { Voting Mechanisms } \\
\text { Development Tools } \\
\text { Creativity Tools }\end{array}$ & $\begin{array}{l}\text { Social Competition } \\
\text { Anarchic Gameplay } \\
\text { Challenges }\end{array}$ & $0.379^{* *}$ & $16 \%$ \\
\hline Player & $\begin{array}{l}\text { Points } \\
\text { Rewards or Prizes } \\
\text { Leaderboards } \\
\text { Badges or Achievements } \\
\text { Virtual Economy } \\
\text { Lotteries or Chance }\end{array}$ & $0.383^{* *}$ & $\begin{array}{l}\text { Points } \\
\text { Rewards or Prizes } \\
\text { Leaderboards } \\
\text { Badges or Achievements } \\
\text { Virtual Economy } \\
\text { Levels or Progression } \\
\text { Collection and Trading }\end{array}$ & $\begin{array}{l}\text { Social Comparison } \\
\text { Social Competition } \\
\text { Social Discovery } \\
\text { Anonymity } \\
\text { Challenges } \\
\text { Certificates } \\
\text { Quests }\end{array}$ & $0.420^{* *}$ & $10 \%$ \\
\hline Overall & & & & & & $9 \%$ \\
\hline
\end{tabular}

Table 7. Bivariate correlation coefficients (Kendall's $\tau$ ) and significance between the Hexad user types and the suggested game design elements for each user type, as well as the percentage of improvement of the new suggestions over the previous ones.

applications because a user's subscale scores predict their preference of different design elements. In addition, we suggested an improved association table between user types and design elements based on the analysis of the correlation coefficients (see Table 7). These results also pointed out to a need for further studies regarding the Philanthropist user type. Its reliability scores were high $(>0.80)$, which means it is accurately measuring a user personality trait. However, we did not found correspondence to a user's preference of difference game design elements.

\section{LIMITATIONS AND FUTURE WORK}

This paper represents the first step toward a standard survey that assesses user preferences for personalization of gamified systems. For this first step, we acquired data from a limited sample to provide a survey validation. Although the sample was large enough for statistical analysis, it was limited to students of one University, which limits generaliza- tion of the survey to a general population. Thus, our next step will be to repeat this study with a larger sample, including people from different cultural origins and a broader age range to validate the model and survey for the general population. In addition, our reliability analysis revealed a need for improving the Player subscale, which we plan to execute on the next survey iteration. Furthermore, we employed a short scale (BFI-10) to assess participants' personality traits. Since short scales are known to potentially have problems with acquiescence, our results should be validated in future work using a more reliable BFI scale.

Our analysis also suggested some unexpected overlap between the Hexad user types and correlations between user types and personality traits, which we plan to investigate further. Finally, we plan to better understand the impact of the Philanthropist user type in user preference towards gamified systems, which our study did not reveal. 


\begin{tabular}{|c|c|c|c|c|c|c|c|c|c|}
\hline \multirow[b]{2}{*}{ User Type } & \multirow[b]{2}{*}{ Item } & \multirow{2}{*}{$\begin{array}{r}\text { 5-items subscale } \\
\text { correlation }(r)\end{array}$} & \multirow{2}{*}{$\begin{array}{r}\text { 4-items subscale } \\
\text { correlation }(r)\end{array}$} & \multicolumn{6}{|c|}{ Rotated Factor Loads } \\
\hline & & & & $1(\mathrm{~A})$ & $2(P)$ & 3 (S) & 4 (D) & $5(\mathrm{R})$ & $6(F)$ \\
\hline \multirow{5}{*}{ Philanthropist (P) } & P1 & 0.786 & 0.780 & & 0.418 & 0.321 & & & 0.468 \\
\hline & P2 & 0.779 & 0.775 & & 0.980 & & & & \\
\hline & $\mathbf{P 3}$ & 0.733 & 0.783 & & 0.437 & & & & 0.450 \\
\hline & P4 & 0.771 & 0.763 & & 0.457 & 0.338 & -0.228 & & \\
\hline & P5 & 0.667 & removed & & & & & & \\
\hline \multirow{5}{*}{ Socialiser (S) } & S1 & 0.730 & 0.734 & & & 0.829 & & & \\
\hline & S2 & 0.624 & 0.617 & & & 0.541 & & 0.201 & \\
\hline & S3 & 0.670 & 0.676 & & & 0.829 & & & \\
\hline & S4 & 0.688 & 0.662 & & & 0.620 & & & \\
\hline & S5 & 0.569 & removed & & & & & & \\
\hline \multirow{5}{*}{ Free Spirit $(\mathrm{F})$} & F1 & 0.529 & 0.480 & & & & & & 0.514 \\
\hline & F2 & 0.491 & 0.546 & & & & 0.383 & & 0.220 \\
\hline & F3 & 0.507 & 0.525 & 0.319 & & 0.352 & 0.353 & & \\
\hline & F4 & 0.538 & 0.496 & & & & & & 0.622 \\
\hline & F5 & 0.373 & removed & & & & & & \\
\hline \multirow{5}{*}{ Achiever (A) } & A1 & 0.603 & 0.574 & 0.359 & & & & 0.222 & \\
\hline & $\mathbf{A 2}$ & 0.483 & 0.485 & 0.288 & & & & 0.395 & \\
\hline & A3 & 0.553 & 0.569 & 0.434 & & & & 0.211 & \\
\hline & A4 & 0.612 & 0.604 & 1.042 & & & & & \\
\hline & A5 & 0.454 & removed & & & & & & \\
\hline \multirow{5}{*}{ Disruptor (D) } & D1 & 0.579 & 0.588 & & & & 0.671 & & \\
\hline & D2 & 0.451 & 0.398 & & & & 0.555 & & 0.249 \\
\hline & D3 & 0.569 & 0.569 & & & & 0.620 & & \\
\hline & D4 & 0.523 & 0.577 & & & -0.213 & 0.732 & & \\
\hline & D5 & 0.323 & removed & & & & & & \\
\hline \multirow{5}{*}{ Player (R) } & R1 & 0.445 & 0.459 & & & 0.318 & & 0.521 & \\
\hline & $\mathbf{R 2}$ & 0.561 & 0.622 & & & 0.255 & & 0.670 & \\
\hline & $\mathbf{R 3}$ & 0.359 & 0.313 & & & & & 0.304 & \\
\hline & R4 & 0.580 & 0.568 & & & & & 0.668 & \\
\hline & $\mathbf{R 5}$ & 0.305 & removed & & & & & & \\
\hline
\end{tabular}

Table 8. Corrected item-total correlations $(r)$ and rotated factor loads $(>=0.20)$ for each of the Hexad survey items.

\section{SUMMARY AND CONCLUSION}

We have presented and validated a standard scale to score users' preferences regarding the six different motivations to use a gameful system according to the Hexad framework: Philanthropist, Socialiser, Free Spirit, Achiever, Disruptor, and Player. The final Hexad scale is composed of 24 items, which together can accurately describe user preferences. Using our survey is more effective than asking users about design elements directly because the survey's goal is to understand more about user psychology in a gamified context than just which game elements they prefer. Furthermore, users are not necessarily gamers and may therefore not be aware of their game preferences and not familiar with game design vocabulary. Therefore, our survey aims to use a common vocabulary. Moreover, correlation analysis of the Hexad user types with different game design elements confirmed the usefulness of the Hexad model as a measure of preferred design elements. This allowed us to suggest a table of game design elements for each user type.
The Hexad User Types framework can be seen as a valid model for personalizing gamified applications after this study. Thus, the survey developed in this paper makes a significant contribution to the areas of HCI and gamification because it will enable researchers and designers to accurately measure user preference for different elements in gameful design.

\section{ACKNOWLEDGMENTS}

Gustavo Tondello would like to thank the University of Waterloo and the CNPq, Brazil for funding his research. Rina Wehbe would like to thank the Cheriton School of Computer Science, University of Waterloo, and NSERC for funding her research. This research has received funding from NSERC (RGPIN-418622-2012), SSHRC (895-20111014, IMMERSe), and the project GEMPLAY (contract no. 844845), funded by the Austrian Research Promotion Agency. The authors would like to thank Elisa Mekler, Melissa Stocco, and the reviewers for providing feedback. 


\section{REFERENCES}

1. Sander Bakkes, Chek Tien Tan, and Yusuf Pisan. 2012. Personalised gaming. Proceedings of The $8^{\text {th }}$ Australasian Conference on Interactive Entertainment Playing the System - IE '12, ACM, 1-10. http://doi.org/10.1145/2336727.2336731

2. Gabriel Barata, Sandra Gama, Joaquim A.P. Jorge, and Daniel J.V. Gonçalves. 2014. Relating gaming habits with student performance in a gamified learning experience. Proceedings of the first ACM SIGCHI Annual Symposium on Computer-Human Interaction in Play - CHI PLAY'14, ACM, 17-25. http://doi.org/10.1145/2658537.2658692

3. Richard Bartle. 1996. Hearts, Clubs, Diamonds, Spades: Players who suit MUDs. Journal of MUD Research 1, 1.

4. Richard Bartle. 2005. Virtual Worlds: Why People Play. Massively Multiplayer Game Development 2, 1.

5. Chris Bateman, Rebecca Lowenhaupt, and Lennart E Nacke. 2011. Player Typology in Theory and Practice. Proceedings of DiGRA 2011.

6. Chris Mark. Bateman and Richard Boon. 2006. $21^{\text {st }}$ Century Game Design (Game Development Series). Charles River Media.

7. Max V. Birk, Dereck Toker, Regan L. Mandryk, and Cristina Conati. 2015. Modeling Motivation in a Social Network Game Using Player-Centric Traits and Personality Traits. Proceedings of User Modeling, Adaptation and Personalization, Springer, 18-30. http://doi.org/10.1007/978-3-319-20267-9_2

8. Marc Busch, Elke Mattheiss, Rita Orji, Peter Fröhlich, Michael Lankes, and Manfred Tscheligi. 2016. Player Type Models - Towards Empirical Validation. Proceedings of the 2016 CHI Conference Extended Abstracts on Human Factors in Computing Systems CHI EA '16, ACM, 1835-1841. http://doi.org/10.1145/2851581.2892399

9. Marc Busch, Elke Mattheiss, Rita Orji, et al. 2015. Personalization in Serious and Persuasive Games and Gamified Interactions. Proceedings of the 2015 Annual Symposium on Computer-Human Interaction in Play - CHI PLAY'15, ACM, 811-816. http://doi.org/10.1145/2793107.2810260

10. Edward L Deci and Richard M Ryan. 1985. Intrinsic Motivation and Self-Determination in Human Behavior. Plenum, New York and London.

11. Edward L. Deci, Haleh Eghrari, Brian C. Patrick, and Dean R. Leone. 1994. Facilitating Internalization: The Self-Determination Theory Perspective. Journal of Personality 62, 1: 119-142. http://doi.org/10.1111/j.1467-6494.1994.tb00797.x

12. Sebastian Deterding, Dan Dixon, Rilla Khaled, and Lennart E Nacke. 2011. From Game Design Elements to Gamefulness: Defining "Gamification."
Proceedings of the $15^{\text {th }}$ International Academic MindTrek Conference: Envisioning Future Media Environments - MindTrek '11, ACM, 9-15. http://doi.org/10.1145/2181037.2181040

13. Lisa Diamond, Gustavo F. Tondello, Andrzej Marczewski, Lennart E. Nacke, and Manfred Tscheligi. 2015. The HEXAD Gamification User Types Questionnaire : Background and Development Process. Workshop on Personalization in Serious and Persuasive Games and Gamified Interactions.

14. Dan Dixon. 2011. Player Types and Gamification. CHI 2011 Workshop Gamification Using Game Design Elements in NonGame Contexts, 12-15.

15. Lauren S. Ferro, Steffen P. Walz, and Stefan Greuter. 2013. Towards personalised, gamified systems: an investigation into game design, personality and player typologies. Proceedings of the $9^{\text {th }}$ Australasian Conference on Interactive Entertainment: Matters of Life and Death - IE '13, 1-6. http://doi.org/10.1145/2513002.2513024

16. Andrew R. Gilpin. 1993. Table for Conversion of Kendall's Tau to Spearman's Rho Within the Context of Measures of Magnitude of Effect for MetaAnalysis. Educational and Psychological Measurement 53, 1: 87-92. http://doi.org/10.1177/0013164493053001007

17. Juho Hamari, Jonna Koivisto, and Harri Sarsa. 2014. Does gamification work? - A literature review of empirical studies on gamification. Proceedings of the $47^{\text {th }}$ Annual Hawaii International Conference on System Sciences, 3025-3034. http://doi.org/10.1109/HICSS.2014.377

18. Juho Hamari and Janne Tuunanen. 2014. Player types: A meta-synthesis. Transactions of the Digital Games Research 1, 2. Retrieved April 10, 2016 from http://todigra.org/index.php/todigra/article/view/13

19. David Howell. 2012. Statistical Methods for Psychology. Cengage Learning.

20. Veronika Huta and Alan S. Waterman. 2014. Eudaimonia and Its Distinction from Hedonia: Developing a Classification and Terminology for Understanding Conceptual and Operational Definitions. Journal of Happiness Studies 15, 6: 1425-1456. http://doi.org/10.1007/s10902-013-9485-0

21. Yuan Jia, Bin Xu, Yamini Karanam, and Stephen Voida. 2016. Personality-targeted Gamification: A Survey Study on Personality Traits and Motivational Affordances. Proceedings of the 34th Annual ACM Conference on Human Factors in Computing Systems - CHI '16. http://doi.org/10.1145/2858036.2858515

22. Daniel Johnson and John Gardner. 2010. Personality, Motivation and Video Games. Proceedings of the $22^{\text {nd }}$ Conference of the Computer-Human Interaction Special Interest Group of Australia on Computer- 
Human Interaction - OZCHI '10, 276.

http://doi.org/10.1145/1952222.1952281

23. Daniel Johnson, Peta Wyeth, Penny Sweetser, and John Gardner. 2012. Personality, genre and videogame play experience. Proceedings of the $4^{\text {th }}$ International Conference on Fun and Games - FnG '12, ACM, 117-120. http://doi.org/10.1145/2367616.2367633

24. Maurits Kaptein, Panos Markopoulos, Boris De Ruyter, and Emile Aarts. 2015. Personalizing persuasive technologies: Explicit and implicit personalization using persuasion profiles.

International Journal of Human Computer Studies 77: 38-51. http://doi.org/10.1016/j.ijhcs.2015.01.004

25. Maurits Kaptein, Boris De Ruyter, Panos Markopoulos, and Emile Aarts. 2012. Adaptive Persuasive Systems: A Study of Tailored Persuasive Text Messages to Reduce Snacking. $A C M$ Transactions on Interactive Intelligent Systems 2, 2: 1-25. http://doi.org/10.1145/2209310.2209313

26. Silvia Erika Kober and Christa Neuper. 2013. Personality and Presence in Virtual Reality: Does Their Relationship Depend on the Used Presence Measure? International Journal of Human-Computer Interaction 29, 1: 13-25. http://doi.org/10.1080/10447318.2012.668131

27. Andrzej Marczewski. 2015. User Types. In Even Ninja Monkeys Like to Play: Gamification, Game Thinking \& Motivational Design. CreateSpace Independent Publishing Platform, 69-84.

28. Andrzej Marczewski. 2015. Gamification Mechanics and Elements. In Even Ninja Monkeys Like to Play: Gamification, Game Thinking \& Motivational Design. CreateSpace Independent Publishing Platform, 165177.

29. Andrzej Marczewski. 2015. Marczewski's User Type Test. Gamified UK. Retrieved July 20, 2016 from http://gamified.uk/UserTypeTest2015/user-typetest.php

30. Isabel Briggs Myers. 1962. The Myers-Briggs Type Indicator. Consulting Psychologists Press, Palo Alto, CA.

31. Lennart E Nacke, Chris Bateman, and Regan L Mandryk. 2011. BrainHex: Preliminary Results from a Neurobiological Gamer Typology Survey. In Proceedings of the $10^{\text {th }}$ International Conference on Entertainment Computing - ICEC 2011. Springer Berlin Heidelberg, 288-293. http://doi.org/10.1007/978-3-642-24500-8_31

32. Lennart E Nacke, Chris Bateman, and Regan L Mandryk. 2014. BrainHex: A Neurobiological Gamer Typology Survey. Entertainment Computing 5, 1: 5562. http://doi.org/10.1016/j.entcom.2013.06.002

33. Oded Nov and Ofer Arazy. 2013. Personality-targeted design: theory, experimental procedure, and preliminary results. Proceedings of the 2013

Conference on Computer Supported Cooperative

Work - CSCW'13, ACM, 977-984.

http://doi.org/10.1145/2441776.2441887

34. Rita Orji, Regan L. Mandryk, Julita Vassileva, and Kathrin M. Gerling. 2013. Tailoring persuasive health games to gamer type. Proceedings of the SIGCHI Conference on Human Factors in Computing Systems - CHI '13, 2467-2476.

http://doi.org/10.1145/2470654.2481341

35. Rita Orji, Julita Vassileva, and Regan L. Mandryk. 2014. Modeling the efficacy of persuasive strategies for different gamer types in serious games for health. User Modeling and User-Adapted Interaction 24, 5: 453-498. http://doi.org/10.1007/s11257-014-9149-8

36. Delroy L. Paulhus. 1984. Two-component models of socially desirable responding. Journal of Personality and Social Psychology 46, 3: 598-609. http://doi.org/10.1037/0022-3514.46.3.598

37. Christopher Peterson, Nansook Park, and Martin E. P. Seligman. 2005. Orientations to happiness and life satisfaction: the full life versus the empty life. Journal of Happiness Studies 6, 1: 25-41. http://doi.org/10.1007/s10902-004-1278-z

38. Beatrice Rammstedt and Oliver P. John. 2007. Measuring personality in one minute or less: A 10item short version of the Big Five Inventory in English and German. Journal of Research in Personality 41, 1 : 203-212. http://doi.org/10.1016/j.jrp.2006.02.001

39. Richard M. Ryan, Edward L. Deci, and Maarten Vansteenkiste. 2016. Autonomy and Autonomy Disturbances in Self-Development and Psychopathology: Research on Motivation, Attachment, and Clinical Process. In Developmental Psychopathology, Dante Cicchetti (ed.). Wiley, 1-54. http://doi.org/10.1002/9781119125556.devpsy109

40. Richard M. Ryan and Edward L. Deci. 2000. Selfdetermination theory and the facilitation of intrinsic motivation, social development, and well-being. The American Psychologist 55, 1: 68-78. http://doi.org/10.1037/0003-066X.55.1.68

41. Richard M. Ryan and Edward L. Deci. 2000. Intrinsic and Extrinsic Motivations: Classic Definitions and New Directions. Contemporary Educational Psychology 25, 1: 54-67. http://doi.org/10.1006/ceps.1999.1020

42. Richard M. Ryan, C. Scott Rigby, and Andrew Przybylski. 2006. The motivational pull of video games: A self-determination theory approach. Motivation and Emotion 30, 4: 347-363. http://doi.org/10.1007/s11031-006-9051-8 
43. Niels Winkler, Martin Kroh, and Martin Spiess. 2006. Entwicklung einer deutschen Kurzskala zur zweidimensionalen Messung von sozialer Erwünschtheit. DIW-Diskussionspapiere 579. Retrieved April 10, 2016 from http://www.econstor.eu/handle/10419/18472

44. Yan Xu, Erika Shehan Poole, Andrew D. Miller, et al. 2012. This is not a one-horse race: understanding player types in multiplayer pervasive health games for youth. Proceedings of the ACM 2012 conference on Computer Supported Cooperative Work-CSCW'12, ACM, 843-852.

http://doi.org/10.1145/2145204.2145330

45. Nick Yee, Nicolas Ducheneaut, Les Nelson, and Peter Likarish. 2011. Introverted Elves \& Conscientious Gnomes: The Expression of Personality in World of Warcraft. Proceedings of the Annual Conference on Human Factors in Computing Systems - CHI '11,
ACM, 753-762.

http://doi.org/10.1145/1978942.1979052

46. Nick Yee, Nicolas Ducheneaut, and Les Nelson. 2012. Online gaming motivations scale: development and validation. Proceedings of the 2012 ACM annual conference on Human Factors in Computing Systems CHI '12, ACM, 2803-2806.

http://doi.org/10.1145/2207676.2208681

47. Nick Yee. 2006. Motivations for Play in Online Games. CyberPsychology \& Behavior 9, 6: 772-775. http://doi.org/10.1089/cpb.2006.9.772

48. Virgil Zeigler-Hill and Sean Monica. 2015. The HEXACO model of personality and video game preferences. Entertainment Computing 11: 21-26. http://doi.org/10.1016/j.entcom.2015.08.001 


\begin{tabular}{|c|c|c|c|c|}
\hline User Types & & Items & $\begin{array}{r}5 \text {-items } \\
\text { subscale } \\
\text { correlation }(r)\end{array}$ & $\begin{array}{r}\text { 4-items } \\
\text { subscale } \\
\text { correlation (r) }\end{array}$ \\
\hline \multirow{5}{*}{ Philanthropist } & $\mathrm{P} 1$ & It makes me happy if I am able to help others. & 0.786 & 0.780 \\
\hline & $\mathrm{P} 2$ & I like helping others to orient themselves in new situations. & 0.779 & 0.775 \\
\hline & $\mathrm{P} 3$ & I like sharing my knowledge. & 0.733 & 0.783 \\
\hline & $\mathrm{P} 4$ & The wellbeing of others is important to me. & 0.771 & 0.763 \\
\hline & P5 & I feel good taking on the role of a mentor. & 0.667 & removed \\
\hline \multirow{5}{*}{ Socialiser } & S1 & Interacting with others is important to me. & 0.730 & 0.734 \\
\hline & $\mathrm{S} 2$ & I like being part of a team. & 0.624 & 0.617 \\
\hline & $\mathrm{S} 3$ & It is important to me to feel like I am part of a community. & 0.670 & 0.676 \\
\hline & S4 & I enjoy group activities. & 0.688 & 0.662 \\
\hline & S5 & It is more fun to be with others than by myself. & 0.569 & removed \\
\hline \multirow{5}{*}{ Free Spirit } & $\mathrm{F} 1$ & It is important to me to follow my own path. & 0.529 & 0.480 \\
\hline & $\mathrm{F} 2$ & I often let my curiosity guide me. & 0.491 & 0.546 \\
\hline & F3 & I like to try new things. & 0.507 & 0.525 \\
\hline & F4 & Being independent is important to me. & 0.538 & 0.496 \\
\hline & F5 & I prefer setting my own goals. & 0.373 & removed \\
\hline \multirow{5}{*}{ Achiever } & A1 & I like defeating obstacles. & 0.603 & 0.574 \\
\hline & & $\begin{array}{l}\text { It is important to me to always carry out my tasks com- } \\
\text { pletely. }\end{array}$ & 0.483 & 0.485 \\
\hline & $\mathrm{A} 3$ & $\begin{array}{l}\text { It is difficult for me to let go of a problem before I have } \\
\text { found a solution. }\end{array}$ & 0.553 & 0.569 \\
\hline & A4 & I like mastering difficult tasks. & 0.612 & 0.604 \\
\hline & A5 & Iam very ambitious. & 0.454 & removed \\
\hline \multirow{5}{*}{ Disruptor } & D1 & I like to provoke. & 0.579 & 0.588 \\
\hline & D2 & I like to question the status quo. & 0.451 & 0.398 \\
\hline & D3 & I see myself as a rebel. & 0.569 & 0.569 \\
\hline & D4 & I dislike following rules. & 0.523 & 0.577 \\
\hline & D5 & Ilike to take changing things into my own hands. & 0.323 & removed \\
\hline \multirow{5}{*}{ Player } & $\mathrm{R} 1$ & I like competitions where a prize can be won. & 0.445 & 0.459 \\
\hline & $\mathrm{R} 2$ & Rewards are a great way to motivate me. & 0.561 & 0.622 \\
\hline & $\mathrm{R} 3$ & Return of investment is important to me. & 0.359 & 0.313 \\
\hline & $\mathrm{R} 4$ & If the reward is sufficient I will put in the effort. & 0.580 & 0.568 \\
\hline & R5 & Hook out for my own interests. & 0.305 & removed \\
\hline
\end{tabular}

\section{How to use the scale:}

1. Ask users to rate how well each item describes them in a 7-point Likert scale.

a. Use only the 24 items numbered from 1-4 in each subscale.

b. Items must be presented without identifying the corresponding type and, if possibly, in random order.

2. Separately add the scores of the items corresponding to each subscale. 


\section{APPENDIX B. CORRELATIONS OF THE HEXAD USER TYPES WITH GAME DESIGN ELEMENTS}

\begin{tabular}{|c|c|c|c|c|c|c|c|}
\hline \multirow{2}{*}{$\begin{array}{l}\text { Suggested by } \\
\text { Marczewski }\end{array}$} & \multirow{2}{*}{ Game Element } & \multicolumn{6}{|c|}{ Improved Associations } \\
\hline & & Socialiser & Free Spirit & Achiever & Disruptor & Player & Philanthropist \\
\hline \multirow{4}{*}{ Philanthropist } & Collection and Trading & $.153^{*}$ & $.148^{*}$ & $.172^{*}$ & & $.259^{* *}$ & \\
\hline & Gifting & $.163^{*}$ & & & & $.207^{* *}$ & \\
\hline & Knowledge sharing & $.184^{* *}$ & $.138^{*}$ & & $.167^{*}$ & $.231^{* *}$ & \\
\hline & Administrative roles & & & & $.199^{* *}$ & & \\
\hline \multirow{5}{*}{ Socialiser } & Guilds or Teams & $.179^{* * *}$ & & & $.169^{*}$ & $.192^{*}$ & \\
\hline & Social networks & $.150^{* *}$ & & & $.197^{* *}$ & $.143^{*}$ & \\
\hline & $\begin{array}{l}\text { Social comparison or } \\
\text { pressure }\end{array}$ & $.152^{* *}$ & & & & $.239^{* *}$ & \\
\hline & Social competition & $.216^{* *}$ & $.249^{* *}$ & $.161^{*}$ & $.320^{* *}$ & $.239^{* *}$ & \\
\hline & Social discovery & $.205^{* *}$ & & & $.179^{* *}$ & $.217^{* *}$ & \\
\hline \multirow{6}{*}{ Free Spirit } & Exploratory tasks & & $.352^{* * *}$ & & & $.152^{* *}$ & $.139^{*}$ \\
\hline & Nonlinear gameplay & & $.221^{* *}$ & & & & $.179^{*}$ \\
\hline & Easter eggs & $.137^{*}$ & $.246^{* *}$ & & $.153^{* *}$ & $.162^{*}$ & \\
\hline & $\begin{array}{l}\text { Unlockable or rare } \\
\text { content }\end{array}$ & & $.225^{* *}$ & & & $.149^{*}$ & $.140^{*}$ \\
\hline & Creativity tools & & $.230^{* * *}$ & & $.252^{* *}$ & & \\
\hline & Customization & & $.198^{* * *}$ & & $.136^{* *}$ & $.162^{* *}$ & \\
\hline \multirow{5}{*}{ Achiever } & Challenges & & $.412^{* *}$ & $.463^{* * *}$ & $.207^{* *}$ & $.317^{* *}$ & $.212^{* *}$ \\
\hline & Certificates & $.142^{*}$ & $.200^{* *}$ & $.229^{* *}$ & & $.228^{* *}$ & \\
\hline & Learning & & $.391^{* *}$ & $.215^{* * *}$ & & & \\
\hline & Quests & & $.236^{* *}$ & $.266^{* * *}$ & & $.245^{* *}$ & \\
\hline & $\begin{array}{l}\text { Levels or Progression } \\
\text { Boss battles }\end{array}$ & $.170^{*}$ & $.204^{* *}$ & $.239^{* *}$ & & $.302^{* *}$ & \\
\hline \multirow{6}{*}{ Player } & Points & $.168^{*}$ & $.201^{* *}$ & $.172^{* *}$ & & $.259^{* * *}$ & \\
\hline & Rewards or Prizes & & $.139^{*}$ & $.167^{* *}$ & & $.301^{* *}$ & \\
\hline & Leaderboards & $.199^{*}$ & & & $.170^{* *}$ & $.276^{* *}$ & \\
\hline & $\begin{array}{l}\text { Badges or } \\
\text { Achievements }\end{array}$ & $.164^{*}$ & & $.208^{* * *}$ & & $.271^{* *}$ & \\
\hline & Virtual economy & & & & & $.273^{* *}$ & \\
\hline & $\begin{array}{l}\text { Lotteries or Games of } \\
\text { chance }\end{array}$ & $.148^{*}$ & & & & $.190^{* *}$ & \\
\hline \multirow{5}{*}{ Disruptor } & Innovation platforms & & & & $.302^{* *}$ & $.166^{*}$ & \\
\hline & Voting mechanisms & & & & $.236^{* *}$ & $.138^{*}$ & \\
\hline & Development tools & & & & $.294^{* *}$ & $.144^{*}$ & \\
\hline & Anonymity & & $.318^{* *}$ & $.289^{* * *}$ & & $.211^{*}$ & \\
\hline & Anarchic gameplay & & $.285^{* *}$ & & $.268^{* *}$ & & \\
\hline
\end{tabular}

Notes.

All correlations measured by Kendall's $\tau$. Only significant correlations are shown.

The bold cells in each column mark the new suggestions of game design elements to support each Hexad user type. ${ }^{*} p<0.05$. ${ }^{* *} p<0.01$. 